\title{
Multipath Routing for Balancing the Load in Manet
}

\author{
S.G. Ramesh kumar, N. Vijaya lakshmi
}

\begin{abstract}
Mobile Ad hoc Networks (MANETs) have attracted quite a concentration owing to their extensive potential applications. Load balancing is vital for enhancing performance in MANETs. Routing overload is the major causes of breaking routing performance in the network. To resolve these challenges, this paper intends a novel routing scheme is called Multipath Routing for Balancing the Load (MRBL) in MANET. In this scheme, the relay node is selected based on the received signal strength. During data transmission, the route node is overload due to heavy data transmission. When utilizing the multipath routing strategy. As a result, decreased network overhead and increased the packet delivery ratio. This work is simulated in NS-2 simulator, and the results show that MRBL scheme performs better than the baseline protocol.
\end{abstract}

Keywords: Multipath routing, Network Simulator, Mobile Ad hoc Networks, Load balancing,.

\section{INTRODUCTION}

MANET is utilized for an extensive range of applications, for example, strategic interactions, tragedy release performances, physical condition care and temporary networking. Entire nodes obtain the data transmission of a node within its coverage area owing to disseminate of the wireless medium and Omni-directional antennae. If two mobile nodes are not present in the coverage area when other nodes found among them can send messages that effectively build connected networks [1].

Usually, many routing protocols are planned to depend on single successive path routing strategy, not including the load intensities. When find the substitute path to maintain data communication procedure creates additional overhead and network delay [16-17]. To overcome this problem, we introduce a multipath routing approach to improve wireless performance. This multipath routing strategy makes several routes from sender to receiver, and it is mainly useful to balance the traffic and reduce the network overload in MANETs [2]. The stay of the article is structured as pursues: sector 2 describes the related work. In sect 3, we proposed Multipath Routing for Balancing the Load in MANETs. Sector 4 analysis of the simulation results. Finally, sector 5 presents the conclusions.

Revised Manuscript Received on December 30, 2019.

* Correspondence Author

S.G. Ramesh kumar, Department of Electrical Engineering, Annamalai university, Chidambaram,India. Email: veesgrramesh@gmail.com

N. Vijaya lakshmi, Department of Electrical Engineering, Annamalai university, Chidambaram, India. Email: vijaya_me2007@yahoo.co.in

(C) The Authors. Published by Blue Eyes Intelligence Engineering and Sciences Publication (BEIESP). This is an open access article under the CC BY-NC-ND license (http://creativecommons.org/licenses/by-nc-nd/4.0/)

\section{RELATED WORKS}

Many papers are reviewing introduced routing protocols for MANETs. These protocols explain and examine the common routing schemes introduced for MANETs,[3]. Multipath routing is an appropriate routing protocol for developing communication and better packet delivery and reduces the network delay. This routing increases the bandwidth efficiency with reliability and minimizes the packet losses in the network [4]. In [5] has exposed that shortest path that provides better performance. Weighted Cumulative Expected Transmission Time [6] metric that selects the better routes to offer high bandwidth links and combine of channel variant hops. Though it does not focuses on congestion due to load balancing in the network.

Stable Backbone based Multipath scheme is utilized to enhance the link quality and choose the stable path among nodes in the network. High link Quality, bandwidth, remaining energy, and low mobility nodes are chosen as candidate nodes [7]. In [8] classified the multipath routing protocols by the major condition used to intend. Disseminated channel selection as well as data packet path in a multi-channel with producing a spanning tree; however, it does not focus on load balancing [9]. Joint channel slot routing focuses on accomplishing best channel assignment. In addition, it provides an interference-free connection and routing by fulfilling assured fairness as well as link scheduling [10].

The disjoint multipath routing method predictable as the efficient path strategy in the network. This method enhances the network lifetime and minimizes energy expenditure [11]. QoS-aware Shortest Multipath Source routing enhances network performance. However, it cannot estimate QoS accurately also increases the link failure in the network [12]. Efficient Multipath Routing protocol (EMPR) [13] applies the packet replication method to offer the required data transmission reliability. One time, set of routes are picked out between the revealed routes and multipath route must decide for allocating traffic through the chose paths. Next, the source operates many ways to transmit created network traffic to the destination. However, this scheme cannot reduce network congestion. If the solution is to progress performance needs for throughput, packet delivery, delay and lifetime [14]. A cross layer frame work is described to better data distribution as well as flexible traffic in wireless network [15]. 


\section{PROPOSED SYSTEM}

In this scheme, the MANET consists of $\mathrm{N}$ number of mobile nodes and $\mathrm{V}$ is the rest of contributing multiple paths. $C$ represents the node capacity, and LD represents the load of the node. We calculate the threshold $(\mathrm{TH})$ of node i equation is given below.

Here, ${ }^{\varepsilon}$ is a predefined system parameter and $A^{\prime}$ is an evaluation for the expected load per unit capability that computed equation is given below

$$
E L=\frac{\sum_{i \in N} L D_{i}}{\sum_{i \in N} C_{i}}
$$

If the current load of $\mathrm{i}$ is larger than the threshold, then the node $\mathrm{i}$ transmit the data via multiple paths. This working procedure is given below.

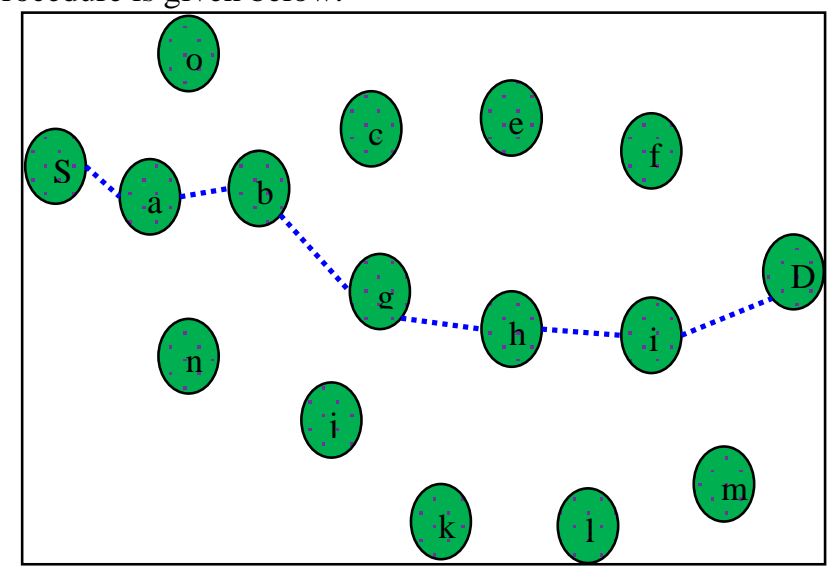

Figure.1 Route Formation in MANET

When a sender nodes desires to communicate a data packet to receiver node $d$, then the source initiates the route-finding from $\mathrm{S}$ to $\mathrm{D}$. The highest received signal strength nodes are elected as route nodes. Next, the source s transmits the data to the selected nodes. Figure 1 illustrates the route formation in the MANET. Here, the source $S$ transmits the data to $d$ via nodes a-b-g-h-j-D.

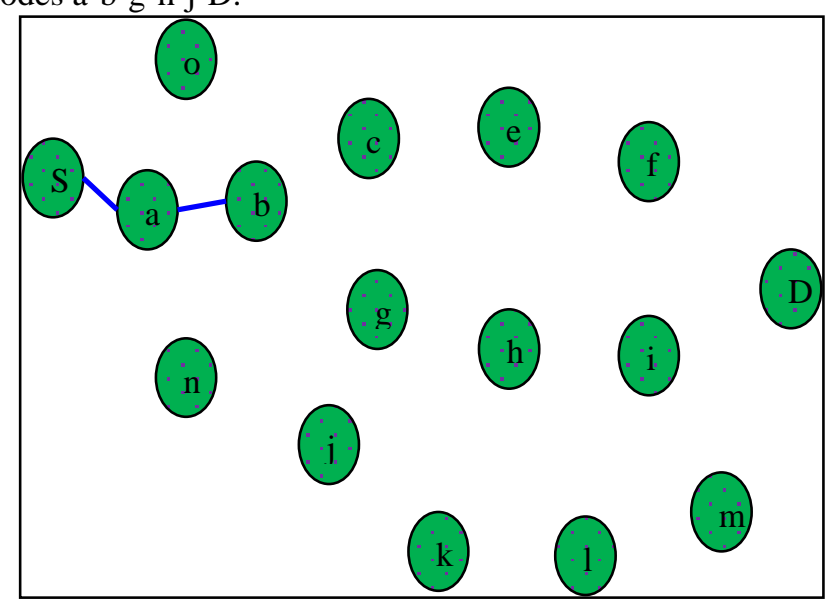

Figure.2 Break the data transmission in MANET

The source successfully passes the data to relay node a and node a pass the data to $b$ next the node $b$ cannot pass the data to $g$ because of heavy load occur in node $b$ is shown in figure 2. The source measures the node b load. If the load is higher

$$
T H_{i}=E L * C_{i}+\varepsilon_{(1)}
$$

compare to the threshold, then make a multipath routing. The node $b$ floods the join request to the neighbour nodes. This function is done till reaches the destination. Then accept the request message nodes are sending the send the join reply to node $b$. Then node $b$ shares the data to near node is illustrated in figure 3.

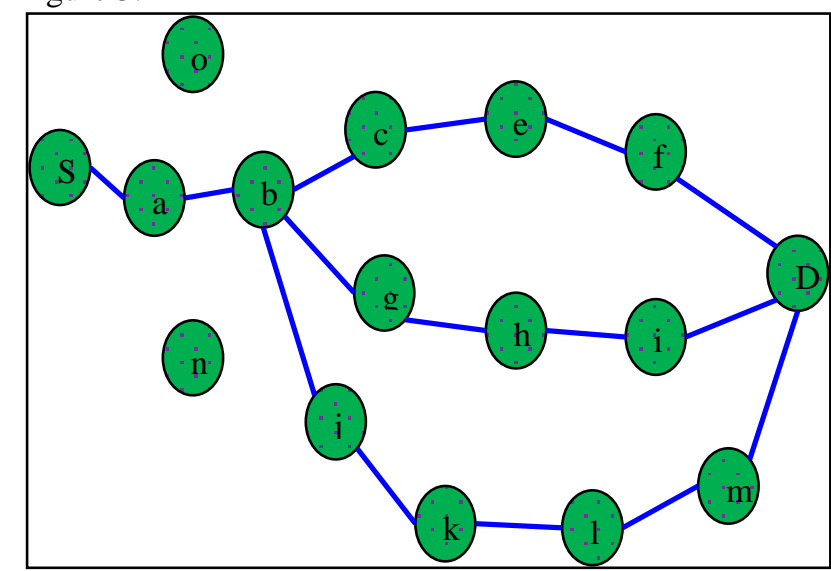

Figure.3 Multipath Routing in MANET

Here the source $S$ transmits the data to destination through relay no desa and b-c-e-f-D, e-h-i-D and i-k-l-m-D. Finally, the destination reaches the data successfully.

\section{RESULT AND DISCUSSION}

The In this section, we estimate the simulation analysis of MRBL protocol using network simulator-2.35. Here, we randomly positioned 50 sensor nodes within an area of $800 \mathrm{~m} \times 800 \mathrm{~m}$. Constant Bit Rate is used for handling the traffic model. The performance of MRBL is analyzed by using parameters routing overhead, and throughput

\subsection{Throughput}

Throughput estimation is normally done for computing the performance of the network, and it is represented as successful deliverance of data rate above a preferred network. It is given in equation 3 ,

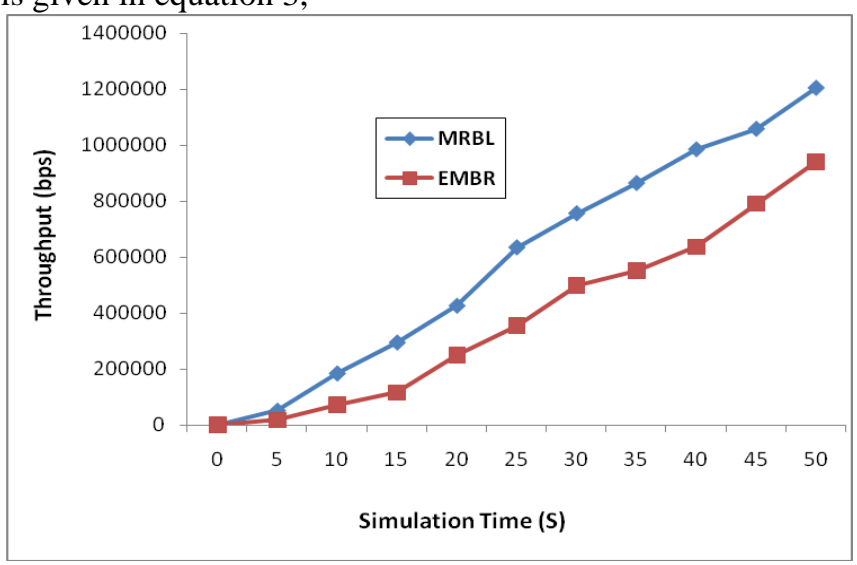

Figure 4 throughput of MRBL and EMPR

Throughput $=\sum_{0}^{\mathrm{n}} \frac{\text { Packet size } * \text { packets received } * 8}{1000}$

Where $\mathrm{n}=$ sensor node count

Figure 4 indicates the proposed method MPRC has greater throughput compared to the existing protocol EMPR.
Blue Eyes Intelligence Engineering \& Sciences Publication

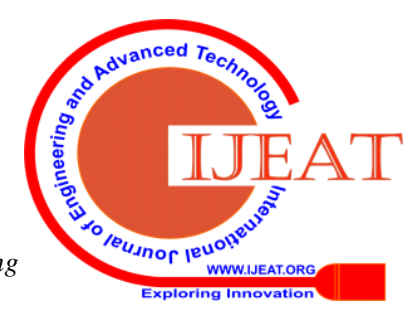




\subsection{Normalized Routing Overhead}

Normalized Routing Overhead (RO) is reported as the amount of control packets (CP) communicated for yielding a data pathway. It is received with computing the fraction among the amounts of CP forward to the amount of CP obtained. Figure 3 represents the RO for EMPR and MRBL.

Figure 5 illustrates the proposed method MRBL has least RO compared to the existing method EMPR in MANET.

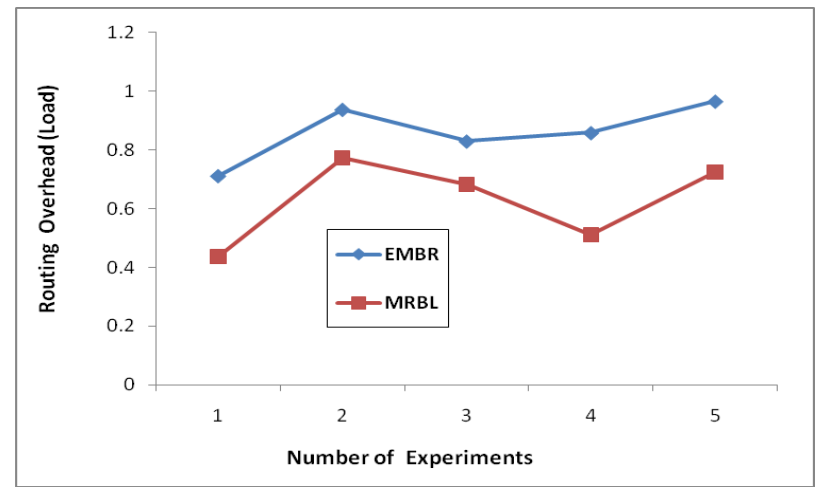

Figure 5 RO of MRBL and EMPR

\section{CONCLUSION}

In this paper, we proposed a Multipath Routing for Balancing the Load (MRBL) in MANET. In this scheme, the relay node is selected based on the received signal strength. During data transmission, the route node is overload due to heavy data transmission. When utilizing the multipath routing strategy. As a result, decreased network overhead as well as increased the throughput efficiency. Simulation results reveal that increases the throughput and reduce the routing overhead in MANETs.

\section{REFERENCES}

1. J. Liu, X. Jiang, X. Nishiyama, N. Kato, " Throughput capacity of MANETs with power control and packet redundancy", IEEE Transactions on Wireless Communications, 2013, vol. 12, no. 6, pp. 3035-3047.

2. X.D.Li, L. Cai, Z. Chen,"Channel Quality and Load Aware Routing in Wireless Mesh Network", IEEE Communication and Networking Conference, 2013, pp. 2068-2073

3. W. Lou, W, Liu, Y. Zhang, "Performance Optimization Using Multipath Routing in Mobile Ad Hoc and Wireless Sensor Networks', Combinator. Optim. Commun. Netw, 2006, Vol. 2, pp.117-146.

4. G. Kalnoor, J. Agarkhed, " QoS based multipath routing for intrusion detection of sinkhole attack in wireless sensor networks", International Conference on Circuit, Power and Computing Technologies, 2016, pp. 1-6.

5. D.D. Couto, D. Aguayo, J. Bicket, R. Morris, “ High-Throughput Path Metric for Multi-Hop Wireless Routing,' Proc. of ACM MOBICOM., 2003.

6. R. Draves, J. Padhye, B. Zill, B, "Comparison of Routing Metrics for Static Multi-Hop Wireless Networks,” Proc of SIGCOMM, 2004.

7. S. Mallapur, S.R. Patil, " Route stability based on demand multipath routing protocol for mobile ad hoc networks", International Conference on Communication and Signal Processing , 2014, pp. 1859-1863.

8. M. Tarique, K.E. Tepe, S. Adibi, S, Erfani, "Survey of Multipath Routing Protocols for Mobile Ad HocNetworks", J. Netw. Comput. Appl. 2009, Vol. 32, pp.1125-1143.

9. A. Raniwala, T. Chiueh, "Architecture and Algorithms for an IEEE 802.11-based Multi-channel Wireless Mesh Network", 2005, Proc of IEEE Infocom.

10. M. Alicherry, R. Bhatia, L. Li, L, "Joint Channel Assigmnet and Routing for Throughput Optimization in Multi-radio Wireless Mesh Netwtorks", Mobicom.
11. A. Liu, Z. Zheng, C. Zhang, Z. Chen, X. Shen, "Secure and energy-efficient disjoint multipath routing for WSNs. IEEE Transactions on Vehicular Technology, 2012, vol. 61, no. 7, pp. 3255-3265.

12.H. Zafar, D. Harle, I. Andonovic, L.Hasan, A. Khattak, "QoS-aware multipath routing scheme for mobile ad hoc networks. International Journal of Communication Networks and Information Security, 2012, vol.4, no.1, pp.1-10.

13.S. Li, R.K. Neelisetti, C.Liu, A.Lim, "Efficient Multi-Path Routing protocol for Wireless Sensor Networks' , Int. J. Wirel. Mobile Netw, 2010, vol 2, pp.110-130.

14.JY. Teo, Y, Ha, CK. Tham, CK, "Interference-Minimized Multipath Routing with Congestion Control in Wireless Sensor Network for High-Rate Streaming", IEEE Trans. Mobile Comput. Mobile Comput. 2008, Vol. 7, pp. 1124-1137.

15. Balaji, K., (2016). Design and Analysis of Increasing throughput and minimising gross layer operations in IEEE 802.11 WLAN, International Research Journal of Engineering and Technology.

16. Balaji, K., (2015). A frame work for integrated routing, scheduling and Traffic Management in MANET, International Research Journal of Engineering and Technology.

17. Shanmugasundaram, T.A., Vijayabaskar, V., "A novel approach for energy efficient clustering in heterogeneous w ireless sensor networks", ARPN Journal of Engineering and Applied Sciences, Vol. 10, No. 5 , 2015, pp. 2172-2176.

\section{AUTHORS PROFILE}

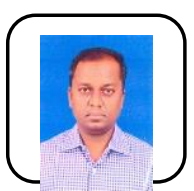

S.G. Ramesh kumar, is working as Assistant Professor in the Department of Electrical Engineering at FEAT, Annamalai University, Chidambaram. He obtained his PG degree from Coimbatore Institute of Technology. Currently he is doing research in the area of wireless sensor networks. He is having 16 years of experience in teaching and research.

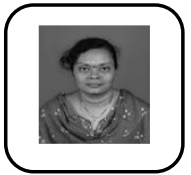

N. Vijaya lakshmi, received her B.E degree in Electrica And Electronics Engineering in 1996 from Annamalai University, India and M.E degree in Power Systems Engineering in 2008 in the same University. She worked as an Assistant Professor in Department of Electrical Engineering, Annamalai University from 2003 to 2016. Currently she is deputed and working as a Lecturer in Govt. Polytechnic college, Coimbatore. 